\title{
A New Approach for Enhancing the Quality of Medical Computerized Tomography Images
}

\author{
Mu'taz Al-Frejat \\ Department of Computer Science \\ Al-Balqa' Applied University \\ Al Salt, Jordan
}

\author{
Mohammad Hjouj Btoush \\ Department of Computer Science \\ Al-Balqa' Applied University \\ Al Salt, Jordan
}

\begin{abstract}
Computerized tomography (CT) images contribute immensely to medical research and diagnosis. However, due to degradative factors such as noise, low contrast, and blurring, CT images tend to be a degraded representation of the actual body or part under investigation. To reduce the risk of imprecise diagnosis associated with poor-quality CT images, this paper presents a new technique designed to enhance the quality of medical CT images. The main objective is to improve the appearance of CT images in order to obtain better visual interpretation and analysis, which is expected to ease the diagnosis process. The proposed technique involves applying a median filter to remove noise from the CT images and then using a Laplacian filter to enhance the edges and the contrast in the images. Also, as CT images suffer from low contrast, a Contrast Limited Adaptive Histogram Equalization transform is also applied to solve this problem. The main strength of this transform is its modest computational requirements, ease of application, and excellent results for most images. According to a subjective assessment by a group of radiologists, the proposed technique resulted in excellent enhancement, including that of the contrast and the edges of medical CT images. From a medical perspective, the proposed technique was able to clarify the arteries, tissues, and lung nodules in the CT images. In addition, blurred nodules in chest CT images were enhanced effectively. Therefore the proposed technique can help radiologists to better detect lung nodules and can also assist in diagnosing the presence of tumours and in the detection of abnormal growths.
\end{abstract}

Keywords-Spatial domain; CT image; Laplacian filter; MedPix database; Lung nodules

\section{INTRODUCTION}

Image processing is a method that is used to perform certain operations on an image to obtain an enhanced image and useful information from the image. It can be described as signal processing, where the input is an image and the output may be an image or characteristic/feature from that image [3]. Image enhancement, also known as filtering, is the most simple and beneficial step in image processing. Sometimes images taken by a satellite or a conventional/digital camera are not clear because of the limitations of the system taking the pictures or the illumination conditions when the picture is taken. In image enhancement, certain image features are accentuated for image analysis or display. However, image enhancement itself does not increase the information present in the image; rather, it enhances the required image characteristics [8]. Enhancement involves modifying the attributes of an image to make it more suitable than the original for a certain observer and a specific activity. Image enhancement mainly includes contrast and intensity manipulation, background removal, noise reduction, filtering, and edge enhancement [15]. In the medical domain, a computerized tomography (CT) scan image is a detailed digital representation or picture of cross-sections of the body created through the use of X-rays. The images are created by using a CT scanner that produces a beam of X-rays while rotating around the body as a computer generates separate images, known as slices, of the body area under examination. The images are then processed by the computer for storage, printing on a film or viewing on a monitor. Computerized tomography reveals a lot of information about the human body through the cross-sectional images it produces and this technique has contributed immensely to medical research and diagnosis. However, due to degradative factors such as noise, low contrast, and blurring, a CT image tends to be a degraded representation of the original body or part under investigation. These factors are caused by the use of a low radiation dose and inappropriate or poor enhancement or restoration algorithms. Also, noise leads to low-contrast details being present in CT images, which causes poor visibility and lack of clarity in the images [2].

To reduce the risk of imprecise diagnosis associated with poor-quality CT images, there is a need to improve the visual detail of CT images and thus aid medical practitioners such as radiologists in making more informed diagnoses by addressing the issues of contrast enhancement and feature enhancement. Image enhancement makes it easier to interpret the results through improving visualization. Several techniques are available to reduce the noise, enhance the edges, and improve the contrast in images [6]. The main objective of this paper is to improve the appearance of medical CT-scan images in order to obtain better visual interpretation and analysis, which is expected to ease the diagnostic process.

Many approaches can be employed to enhance a digital image without destroying it and they can be categorized into two main types: spatial domain and frequency domain methods. Spatial domain methods directly deal with the pixels of the image to obtain the desired enhancement, whereas frequency domain methods involve initially transferring the image into a frequency domain so that the image's Fourier transform is computed first. All the enhancement operations are done on the Fourier transform of the image, after which an Inverse Fourier transform is performed to obtain the intended final image [11]. 
The main operations that can be applied to process digital images are [20].

1) The point operation, where the result of processing a pixel depends only on its value.

2) The local operation, where the result of processing a pixel depends on the values of its neighbourhood. The neighbourhood is a finite set of pixels around the pixel being processing. Defining the neighbourhood is an important part of modern digital image processing and the most common neighbourhoods are 4-connected and 8-connected for rectangular sampling and 6-connected for hexagonal sampling (see Fig. 1).

3) The global operation, where the result of processing a specific coordinate depends on the value of all the coordinates in the input image.

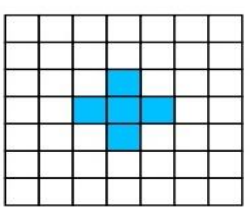

(a)

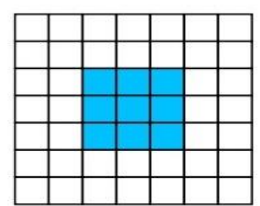

(b)

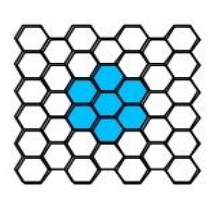

(c)
Fig. 1. Representation of commonly used neighbourhoods: (a): 4-connected neighbourhood; (b): 8-connected neighbourhood; (c): 6-connected neighbourhood [20]

Image quality is the characteristic of an image that is based on the visible image degradation. Imaging systems introduce a quantity of artifacts or distortion to the images produced. The factors that affect image quality are [1]:

a) Blur: Sometimes the boundary of the organs or the lesion is sharp but the image is not sharp. This can occur due to geometric blur or lack of sharpness of the object or receptor.

b) Noise: Uncertainty or lack of precision in the recording of a signal can occur due to the presence of fewer photons, of grains in the radiographic film, intensifying screens with large grains, and the electronic noise of the detector or amplifier.

c) Contrast: Image contrast refers to the minute differences in the brightness between two regions in an image, which can be affected by tissue thickness and density, electron density in the tissue, X-ray energy $(\mathrm{kV})$, X-ray spectrum, scatter rejection, windowing level of CT and digital subtraction angiography, film characteristics, and screen characteristics.

d) Distortion and artifacts: The inaccurate recording of the real size, shape, and relative positions of the items in an image may be due to the visualization of grid on the film, dust on the screen, bad contact of the film with the screen, and bad positioning of the patient. The motion artifact appears when there is patient motion during the acquisition of the CT image, where even a small movement will cause blurring of the image.

\section{LITERATURE REVIEW}

Image enhancement is an important aspect of medical image processing because it can assist in correct diagnosis and treatment planning. Numerous articles have been published on this topic and some of the works most relevant to the technique proposed in this paper are discussed here.

Bhardwaj and Singh [5] address the problem of the low contrast in and poor quality of medical images by proposing a process to enhance medical images based on the Haar transform, soft thresholding, and a nonlinear approach for contrast enhancement. The problem with using the Haar wavelet transform lies in finding a suitable way to extract high-frequency information and to decompose high-frequency sub-images of wavelets. However, the approach proposed by the authors helps in the effective extraction of high-frequency information because the experimental results showed that the proposed algorithm not only improves image contrast, but also preserves the original image's edge property. The final result is good visibility, which is essential for accurate medical diagnosis.

Rajput et al [17] propose the use of nonlinear enhancement techniques such as the discrete wavelet transform and histogram equalization to improve the contrast in medical images. These techniques together improve the lower and the higher contrast areas of an image in both the spatial and frequency domain. Different parameters such as the mean square error, peak signal, and noise ratio are used to evaluate the effectiveness of the proposed method. The results indicated that the peak signal is a better parameter for the pre processed histogram technique than the mean square error or noise ratio. The results also proved that the wavelet transform is better than the simple histogram technique. The discrete wavelet transform enhances the image edges, which implies that it can mainly be used for de-noising images. Based on the experiments performed, the algorithm proposed by the authors can be used to enhance contrast in the image while preserving the quality of the image.

Ritika [16] proposes a novel approach to enhance the contrast of medical images with the help of morphology. The method uses a multiscale structuring element, where white and black (bright and dark) regions are extracted for various scales of the image that correspond to different scales of the structuring element. Then these features are combined with the original image to produce a final image with enhanced contrast. The author's proposed algorithm was implemented and executed on a set of greyscale medical images including a synthetic image obtained through the Monte Carlo simulation method. A comparison of the results of the proposed approach with those obtained from existing methods for contrast enhancement showed that the proposed approach gives better results and makes the features of the images clearer, but there is a slight increase in noise. However, this drawback could be reduced through further research and the algorithm could be further extended through the use of non-flat structuring elements. 
Hirani and Totsuka [7] developed a hybrid technique based on the concept of Projection on Convex Sets (POCS) to reduce noise in images caused by a mixture of types of noise. The approach works by searching for noisy pixels so as to replace them with pixels from the neighbourhood. This is because the algorithm can be able to handle images that feature varying intensities. The method was shown to be quite useful in the reconstruction of images that show repeating patters. However, this method showed challenges in that the proposed technique is that the contents of repair images and the sample have to be approximately translated versions of each other.

\section{MATERIALS AND METHODS}

The technique proposed in this paper consists of three main steps:

1) A median filter for noise reduction:

2) A Laplacian filter under an eight neighbour (-8 in centre) mask for edge enhancement; and

3) A Contrast Limited Adaptive Histogram Equalization (CLAHE) transform for contrast enhancement.

The proposed technique was implemented using Matlab language. See Appendix A

\section{A. Median Filter}

The median represents a partitioning value between the higher weights and lower weights. It is categorized as an order-statistic filter. The filter uses the median value for each pixel by using a sliding kernel around it. If the kernel is square, then there is an odd number of cells in the kernel and picking the median value is easy. If the kernel is not square, then the mean value from the two contesting cells is used [10].

In image processing, the median filter is used extensively to replace unwanted pixel values with a more suitable median value from the surroundings. It is a nonlinear filtering technique that is often used to reduce noise. This noise reduction technique is often used as a preprocessing step to improve the results of image processing. The median filter often gives better results than a mean or average filter. Moreover, it can be used to remove Gaussian noise as well as pepper and salt noise [9].

Like the mean filter, an $\mathrm{n} \times \mathrm{n}$ filter is used to replace the pixel value with its median according to the values of its neighbouring pixels. Instead of replacing the pixel value with its mean, it replaces the central value with the median value. Filtering is applied to each pixel of the image separately with help of an $\mathrm{n} \times \mathrm{n}$ filter kernel. To calculate the median value, pixel values are sorted in ascending order to find the number that has an equal number of values before and after it. The central pixel value is then replaced by the calculated median value [13]. Figure 2 provides an example of the application of the median filter on a pixel using a 3 x 3 kernel size. From the figure,

The pixel values in ascending order are
$6,9,11,15,19,20,24,32,34$ and the median $=19$.

\begin{tabular}{|c|c|c|}
\hline 11 & 15 & 9 \\
\hline 32 & 6 & 34 \\
\hline 19 & 24 & 20 \\
\hline
\end{tabular}

Fig. 2. Part of image source [13]

The median filter is comparatively more robust than the mean filter. In addition, the median filter is more accurate because only one neighbour participates in the final assignment of the central pixel value. As the median value is only one value from the neighbours, it is not affected by the other neighbours at all. This filter preserves edges while removing noise [13].

\section{B. Laplacian Filter (using second-order derivatives in image enhancement)}

The use of second derivatives for enhancement involves defining a discrete formulation of the second-order derivative and then developing a filter mask using that formulation [21] [19]. In this work, the interest is isotropic filters whose response does not depend on the direction of the discontinuities within the image to which the filter is applied. Isotropic filters are described as rotational invariant. According to [6], the simplest isotropic derivative operator is the Laplacian, which is defined (for a function $\mathrm{f}(\mathrm{x}, \mathrm{y})$ of two variables) as [6].

$$
\nabla^{2} f=\frac{\partial^{2} f}{\partial x^{2}}+\frac{\partial^{2} f}{\partial y^{2}}
$$

The Laplacian is a linear operator because the derivatives of any order are linear operations. Equation (1) has to be expressed in discrete form for use in digital image processing. There are several ways to define the digital Laplacian employing neighbourhoods. The definition must satisfy the attributes of the second derivative. As there are two variables, the notation for the partial second-order derivative in the $\mathrm{x}$ direction is employed [6], [21] [19] as follows:

$$
\frac{\partial^{2} f}{\partial x^{2}}=f(x+1, y)+f(x-1, y)-2 f(x, y)
$$

And likewise in the $y$-direction as:

$$
\frac{\partial^{2} f}{\partial y^{2}}=f(x, y+1)+f(x, y-1)-2 f(x, y)
$$

The digytal adoption of the two-dimensional Laplacian in Eq. (1) is obtained by summing the two parts [21]:

$$
\nabla^{2} f=[f(x+1, y)+f(x-1, y)+f(x, y+1)+f(x, y-1)]-4 f(x, y)
$$

Equation (4) can be adopted at all points $(\mathrm{x}, \mathrm{y})$ in an image through convolving the image with the following mask, which provides an isotropic outcome for rotations in increments of 90 degrees [21]. 


\begin{tabular}{|c|c|c|}
\hline 0 & 1 & 0 \\
\hline 1 & -4 & 1 \\
\hline 0 & 1 & 0 \\
\hline
\end{tabular}

Fig. 3. Filter mask used to implement Laplacian [6]

In the definition of the digital Laplacian, the diagonal directions can be integrated by adding two terms to Eq. (4); one term for each of the two diagonal directions. The form of each new term is identical to either Eq. (3) or (2). However, the coordinates are along the diagonals and because each diagonal term also comprises a $-2 \mathrm{f}(\mathrm{x}, \mathrm{y})$ term, the total deducted from the difference term would be $-8 \mathrm{f}(\mathrm{x}, \mathrm{y})$. Figure4 demonstrates the mask employed to adopt this new definition [19].

\begin{tabular}{|c|c|c|}
\hline 1 & 1 & 1 \\
\hline 1 & -8 & 1 \\
\hline 1 & 1 & 1 \\
\hline
\end{tabular}

Fig. 4. Diagonal Laplacian mask [6]

The Laplacian filter is a linear spatial filter that is used to enhance the quality of grey-scale images. This filter is very suitable for reducing noise in an image. In addition, it enhances the linear features of images [21]. The Laplacian is used for image enhancement as follows [22] [19]:

$$
g(x, y)= \begin{cases}f(x, y)-\nabla^{2} f(x, y) & \begin{array}{l}
\text { If the centre coefficient of the } \\
\text { Laplacian mask is negative }
\end{array} \\
f(x, y)+\nabla^{2} f(x, y) & \begin{array}{l}
\text { If the centre coefficient of the } \\
\text { Laplacian mask is positive. }
\end{array}\end{cases}
$$

Figure5 (a) shows a non-contrast axial CT-scan image of a lung with multiple parenchymal nodules. Figures5(b)and 5(c) depict the effects of filtering with a Laplacian mask $\left[\begin{array}{llll}0 & 1 & 0 ; 1\end{array}\right.$ $-41 ; 0$ 1] and a diagonal Laplacian mask [1 11 1; $1 \quad-8$ 1; 1 $11 ;$;,respectively.

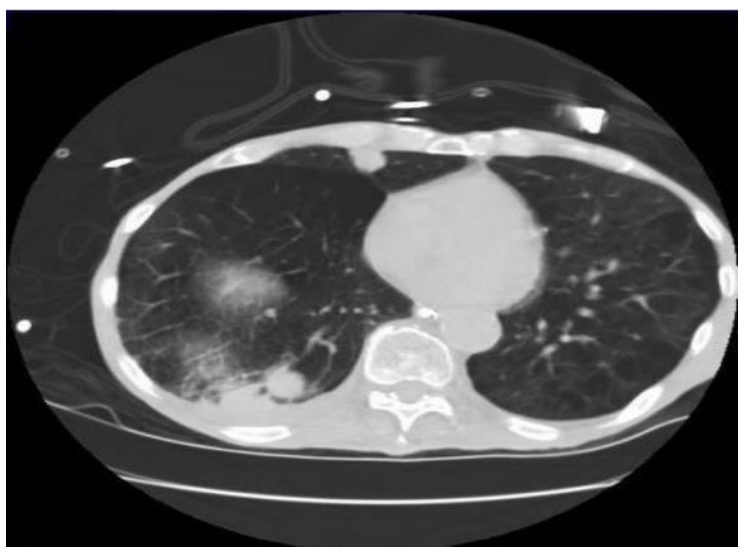

Fig. 5. (a): Lung CT-scan image [12]

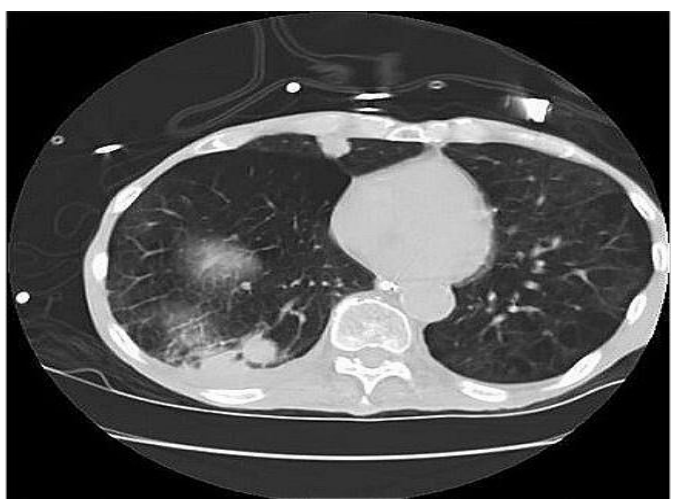

Fig. 5. (b): Enhancement obtained by four-neighbour Laplacian mask (-4 in the centre).

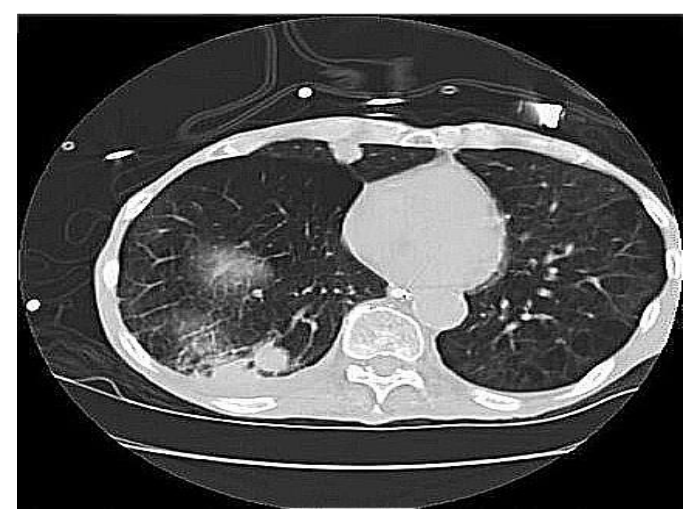

Fig. 5. (c): Enhancement obtained by diagonal Laplacian mask (-8 in the centre)

\section{Contrast Limited Adaptive Histogram Equalization}

Unlike the Adaptive Histogram Equalization (AHE) method, the CLAHE method can be applied to small regions of data known as tiles rather than to the whole image in order to enhance the local contrast in an image. In CLAHE, the eventual neighbouring tiles are then combined by using bilinear interpolation. Also, to avoid noise amplification, the contrast in the homogeneous part can be limited. These specific parts can be characterized by a high peak in the histogram linked with the contextual parts because many pixels fall inside the same grey range. By adopting CLAHE, the slope that comes along with the grey-level assignment scheme is highly limited. This can be possible by only allowing a maximum number of pixels in each particular bin associated with the local histogram [23].

The clip limit is defined as a multiple of the average histogram contents. With a low clip limit, the maximum slope of the local histogram will be low and therefore lead to limited contrast enhancement. A factor of one hinders contrast enhancement; redistribution of histogram bin values can be avoided by using a very high clip limit, which is equivalent to the AHE method. The main strength of the CLAHE transform is its modest computational requirements, ease of application, and excellent results for most images [23].

Figure 6(a) shows an original CT-scan image. Figures 6(b)-(d) depict the effect of CLAHE on the image under different clip limits. 

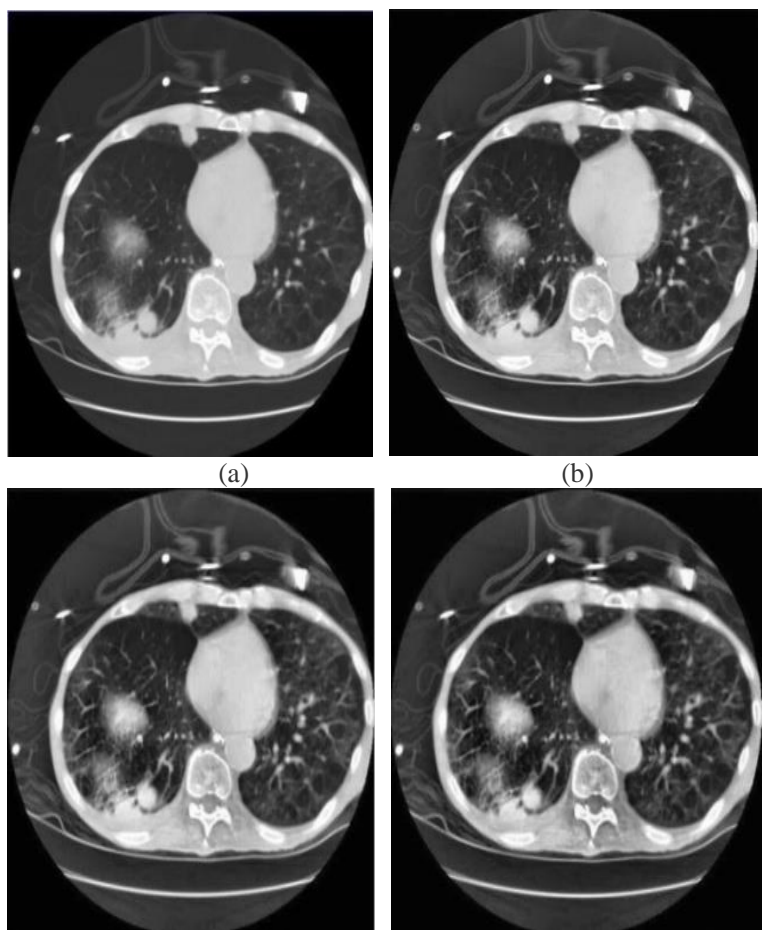

(c)

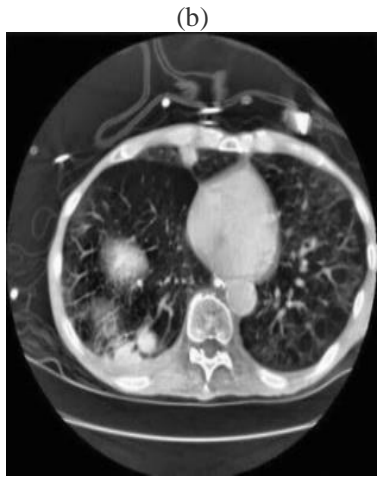

(d)

Fig. 6. The original CT scan image and the results of applying CLAHE Transform (a): The original CT scan image; (b): image at clip limit $=0.002$; (c): image at $0.005 ;(\mathbf{d})$ : image at 0.008

\section{RESLUTS}

This work is focused on enhancing the appearance of the nodules and tumours present in medical CT-scan images of the chest and lungs of human patients. Some results of applying the proposed technique are illustrated in Figures 7-14 below.

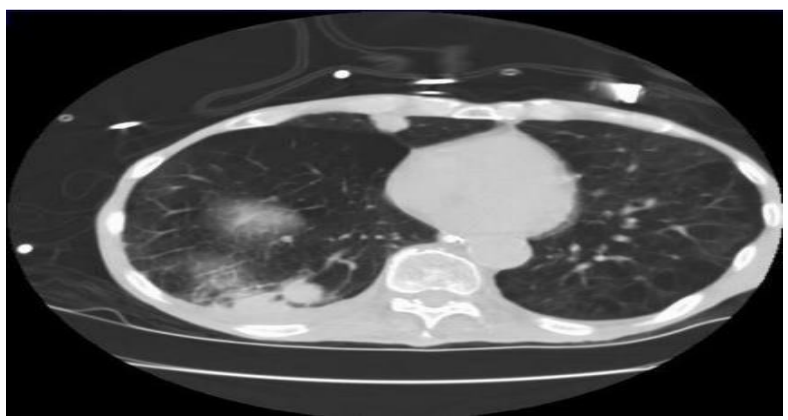

Fig. 7. Lung CT-scan image [12] (Test image \#1)

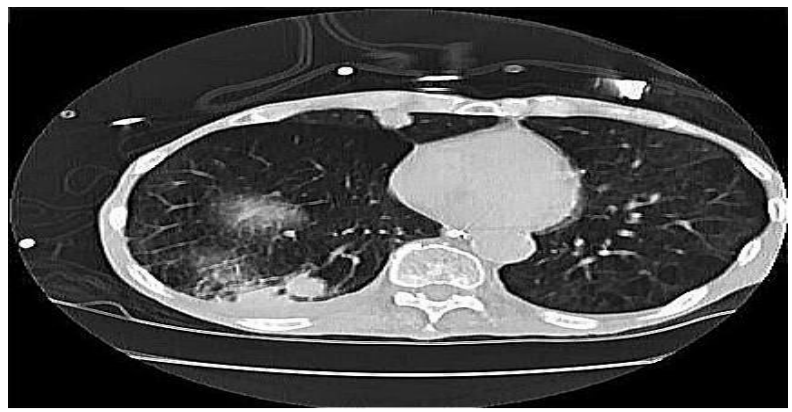

Fig. 8. Enhancement obtained by applying proposed technique to Fig. 7.

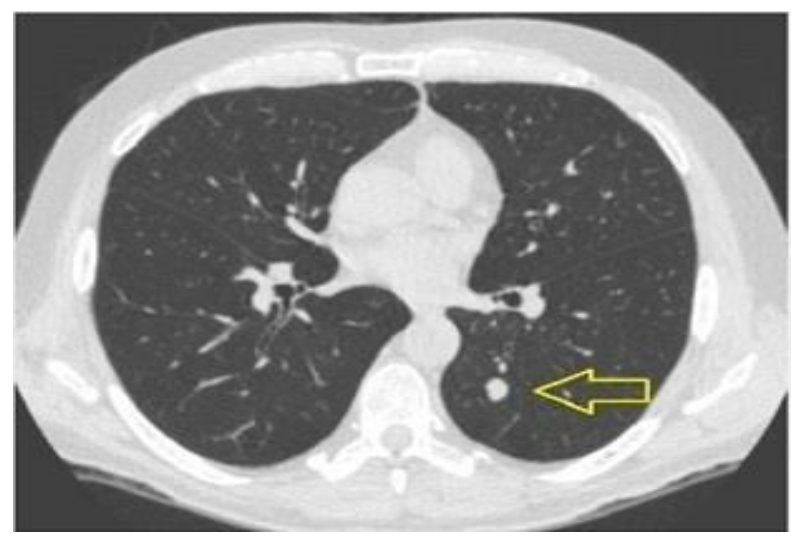

Fig. 9. Lung CT-scan image [12] (Test image \#2)

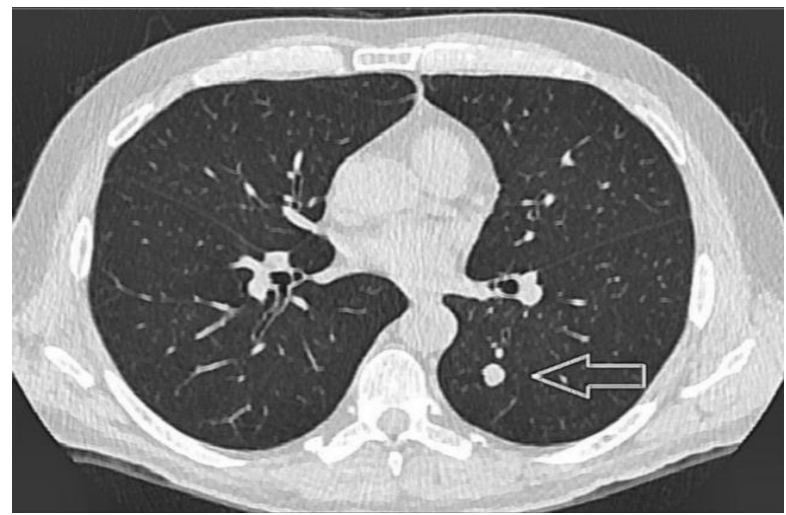

Fig. 10. Enhancement obtained by applying proposed technique to Fig. 9

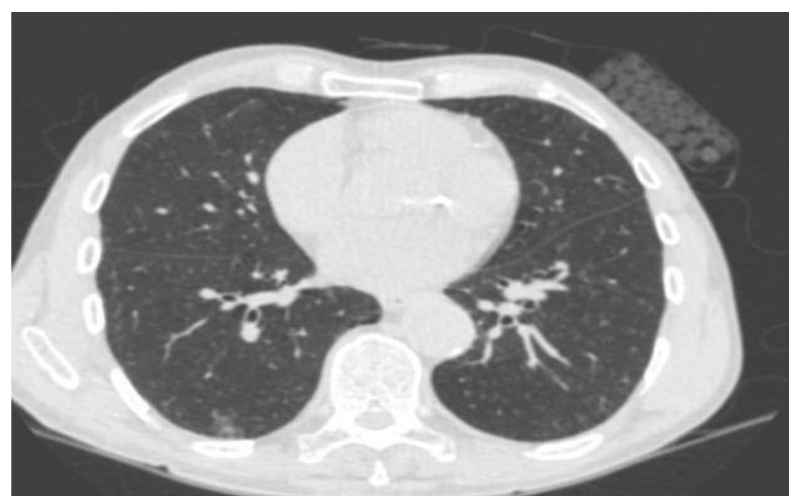

Fig. 11. Lung CT-scan image [12] (Test image \#3)

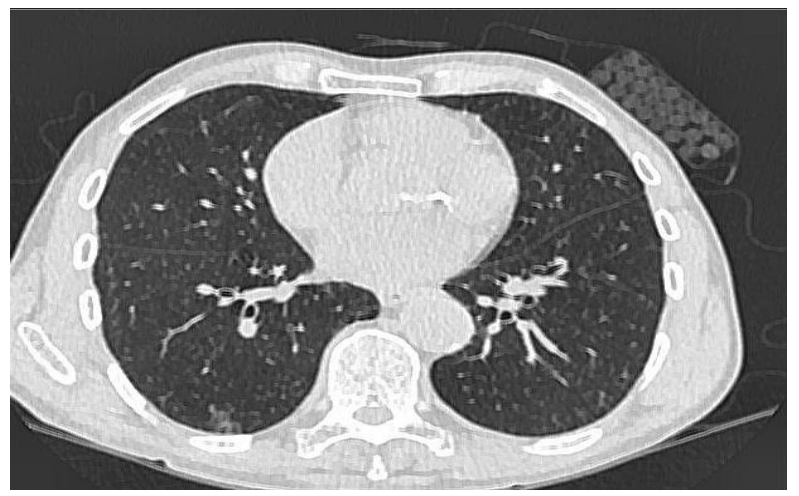

Fig. 12. Enhancement obtained by applying proposed technique to Fig. 11 


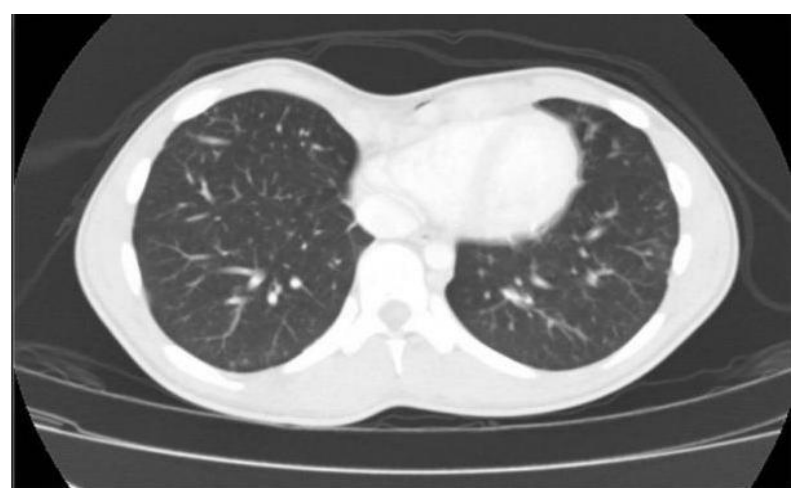

Fig. 13. Lung CT-scan image [12] (Test image \#4)

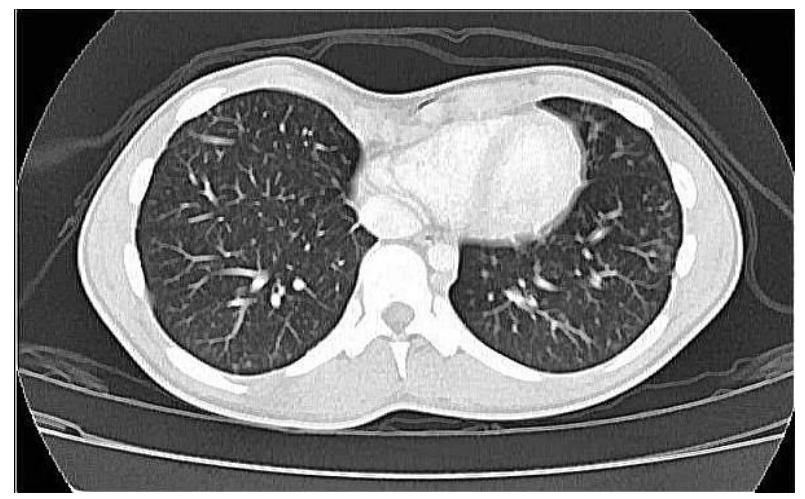

Fig. 14. Enhancement obtained by applying proposed technique to Fig. 13.

\section{DISCUSSION}

Image enhancement is indisputably crucial in medical imaging. It increases contrast, gets rid of noise and blurring, and improves the quality of photographs for human viewers. To enhance medical CT-scan images, the first step of the proposed algorithm addresses the issue of noise reduction by applying a median filter to remove high-frequency components such as salt and pepper noise, Gaussian noise, and impulsive noise. As shown by the figures 8, 10, 12, and 14 in the Results section, the median filter was able to effectively remove above mentioned types of noise medical CT-scan images to improve image clarity.

The second step of the proposed algorithm is the application of a Laplacian filter under a diagonal ( -8 in the centre) mask, which helps in enhancing the edges and the contrast in the CT-scan images. This step is necessary to clarify the details of the image. Edge enhancement by Laplacian filter is useful in the detection and extraction of objects in images. Moreover, edge enhancement with this filter helps in detecting nodules and tumours in CT-scan images. Figures $8,10,12$, and 14 above show that filtering by a Laplacian filter under an 8-neighbour (-8 in centre) mask was able to enhance the edges and contrast effectively. In addition, the details in these images are clearer and sharper. The lung nodules and their surrounding tissue have been enhanced and sharpened effectively, which makes their detection through human visual perception easier.

The third and final step of the proposed algorithm is the use of the CLAHE technique in order to enhance the contrast and to clarify the details in the CT-scan images. In medical applications, contrast enhancement is very important as it improves the perceptibility of the cross-sectional images of body. Moreover, this process increases the brightness variance between the body and its related background. This allows for an accurate analysis of low-contrast CT images. From Figures 8,10 , and 12 above, it can be seen that application of the CLAHE transform was able to enhance the appearance of the tissues and the blood vessels. In addition, new details have appeared.

Thus, it is apparent from the figures in the Results section that the proposed algorithm enhances medical CT-scan images. However, it is also important to seek the opinion of experts regarding the effectiveness of the proposed technique from the medical perspective. While image restoration methods are assessed objectively through the use of mathematical models to test image degradation, when comparing and evaluating the performance of image enhancement techniques it is necessary to judge the visual quality of the enhanced images [18] as perceived by the human eye.

In the context of this work, doctors in hospitals are the best category of human viewer to check if the proposed technique gives a better result to aid them in making the correct diagnosis by comparing the enhanced images with the original images [4]. As this work is targeted at assisting radiologists, they were chosen as image quality assessors. All the enhanced images together with the original images were presented to a group of five specialist radiologists to measure the enhancement percentage. The experts presented percentage ratio for enhancement of images which is $85 \%$ from the original images.

\section{CONCLUSION AND FUTUTRE WORK}

One of the biggest challenges in digital image processing, particularly in computerized tomography, is the occurrence of degradation due to several factors such as noise, blurring, and low contrast, which are associated with various real-world limitations and which corrupt the quality of images. It is important therefore to subject CT images to an image enhancement process to improve their clarity before they are used in the diagnostic process.

The principal goal of image enhancement involves modifying the attributes of an image to make it more suitable than the original image for a certain observer and a specific activity. Image enhancement encompasses manipulation of contrast and intensity, background removal, reduction of noise, filtering, and sharpening of edges to improve quality.

The results presented in this paper show that the proposed technique can enhance medical CT-scan images effectively, and this finding is supported by the results of a subjective assessment by a group of medical experts. Enhancements were made to the contrast and the edges of a range of medical CTscan images. From the medical perspective, the proposed technique clarified the arteries, tissues, and nodules. In addition, blurred nodules were enhanced effectively. Thus the proposed technique can help radiologists in the detection of lung nodules as well as assist in diagnosing the presence of 
tumours and in the detection of abnormal growths. Future work will focus on design a new technique to enhance the medical image quality.

\section{ACKNOWLEDGMENT}

This paper would not have been produced without Allah's aid. The authors would like to thank the radiologists and the staff members at Prince Hamza and Al-Isra hospitals for facilitating part of the assessment. Special thanks also to Doctor Kifah Adib Al-Hafez for his efforts and help.

\section{REFERENCES}

[1] Accra, 2011. Image quality and patient dose. Available at: http://documents.mx/documents/lecture-05-image quality-and-patientdose.html. [Accessed 2015-10-5].

[2] A. Zohair, A.Shamil, and S. Ghazali, "Latest Methods of Image Enhancement and Restoration for Computed Tomography: A Concise Review," Applied Medical Informatics, Vol. 36, Issue 1, pp. 1-12, (2015).

[3] I. A. Ansari and R. Y. Borse, "Image Processing and Analysis “, International Journal of Engineering Research and Applications, Vol.3, issue.4, pages.1655-1658,ISSN.2248-9622,(2013).

[4] P. Aparma, I. Anand, and B. Sanjay, "Contrast Limited Adaptive Histogram with edge enhancement for medical X-ray images," International Journal of Humanities, Arts, Medicine and Sciences, Vol. 3, Issue 6, pp. 9-16, (2015).

[5] A. Bhardwaj and M. Singh, "A novel approach of medical image enhancement based on wavelet transform", International journal of engineering research and application,Vol.2, issue.3, pages.23562360,ISSN.2248-9622,(2012).

[6] R. C. Gonzalez and R. E. Woods, "Digital Image Processing,", New York: Prentice Hall, (2008).

[7] A. N. Hirani and T. Totsuka, "Combining Frequency and Spatial Domain Information for Fast Interactive Image Noise Removal", in Proceedings of the International Conference on Computer Graphics and Interactive Techniques, pages.269-276,(1996).

[8] S. Jayaraman, S.Esakkiranjan and T. Veerakumar, T, "Digital image processing", New Delhi: Tata McGraw Hill,2015.

[9] R. C. Kenneth, "Digital image processing",USA: Prentice Hall.1996

[10] V. Kumar, P. Gupta, "Importance of statistical measures in digital image processing," International Journal of Emerging Technology and Advanced Engineering, Vol. 2, Issue 8, pp. 56-62, (2012).
[11] R. Maini and H. Aggarwal, "A Comprehensive Review of Image Enhancement Techniques ", JOURNAL OF COMPUTING, Vol.2, issue.3, pages.8-13, ISSN.2151-9617, (2010).

[12] MedPix, Medical Image Database, Radiology. At: https://medpix.nlm.nih.gov/home. [Accessed 2015-7-22].

[13] S. Mitra and J.Sicuranza, "Nonlinear image processing,", San Diego: Academia Press, 2001.

[14] S. Mundhada and V. Shandilya, "Spatial and transformation domain techniques for image enhancement", International journal of engineering science and innovative technology, Vol.1, issue.2, pages.213-216, ISSN.2319-5967(2012).

[15] M. Ritika And S. Kaur, "Contrast Enhancement Techniques for ImagesA Visual Analysis," International Journal of Computer Applications, Vol. 64, issue.17, pages.20-25, ISSN.0975 - 8887, (2013).

[16] M. Ritika, "A novel approach of local contrast enhancement of medical images using mathematical morphology", International journal of computer science and information technology and security, Vol.2, issue.2, pages.392-397, ISSN.2249-9555, (2012).

[17] Y. Rajput, V. Rajput, A. Thakur and G. Vyas, "Advanced image enhancement based on wavelet and histogram equalization for medical images", IOSR Journal of electronics and communication engineering, Vol. 2, issue.6, pages.12-16, ISSN.2278-2834, (2012).

[18] H.E. Awad, M. Hjouj Btoush and E. Tadros, "A Robust Watermarking Algorithm Based on Two Dimensional Cellular Automata," International Journal of engineering Research and Industry Applications, Vol. 5, Issue.4, pp. 249-264, (2012).

[19] I. Shakhrah, "Digital High-Pass Filters with Milder High-Pass Effect on Digital Images", American Journal of Engineering and Applied Sciences, Vol.8, issue.3, pages.360-370, ISSN.10.3844, (2015).

[20] I. Suneetha and T. Venkateswarlu, "Enhancement Techniques for True Color Images in Spatial Domain ", International Journal of Computer Science and Technology, Vol.3, issue.2, pages.814-820, ISSN.2229-433, (2015)

[21] K. Thangadurai. And K. Padmavathi, "Noise Reduction and Image Sharpening Using Linear Spatial Filtering in Plant Leaves Disease Detection ", Journal Computer Technology \& Applications, Vol.5, issue.4, pages.1561-1565, ISSN.2229.6093, (2014).

[22] A. M. Trifas, Medical image enhancement. PhD Dissertation, Louisiana State University, East Lansing, MI, USA.2005.

[23] K. Zuiderveld, "Contrast Limited Adaptive Histograph Equalization," In Graphics gems IV, Paul S. Heckbert (Ed.). Academic Press Professional, Inc., San Diego, CA, USA, 1994, pp. 474-485. 
APPENDIX A

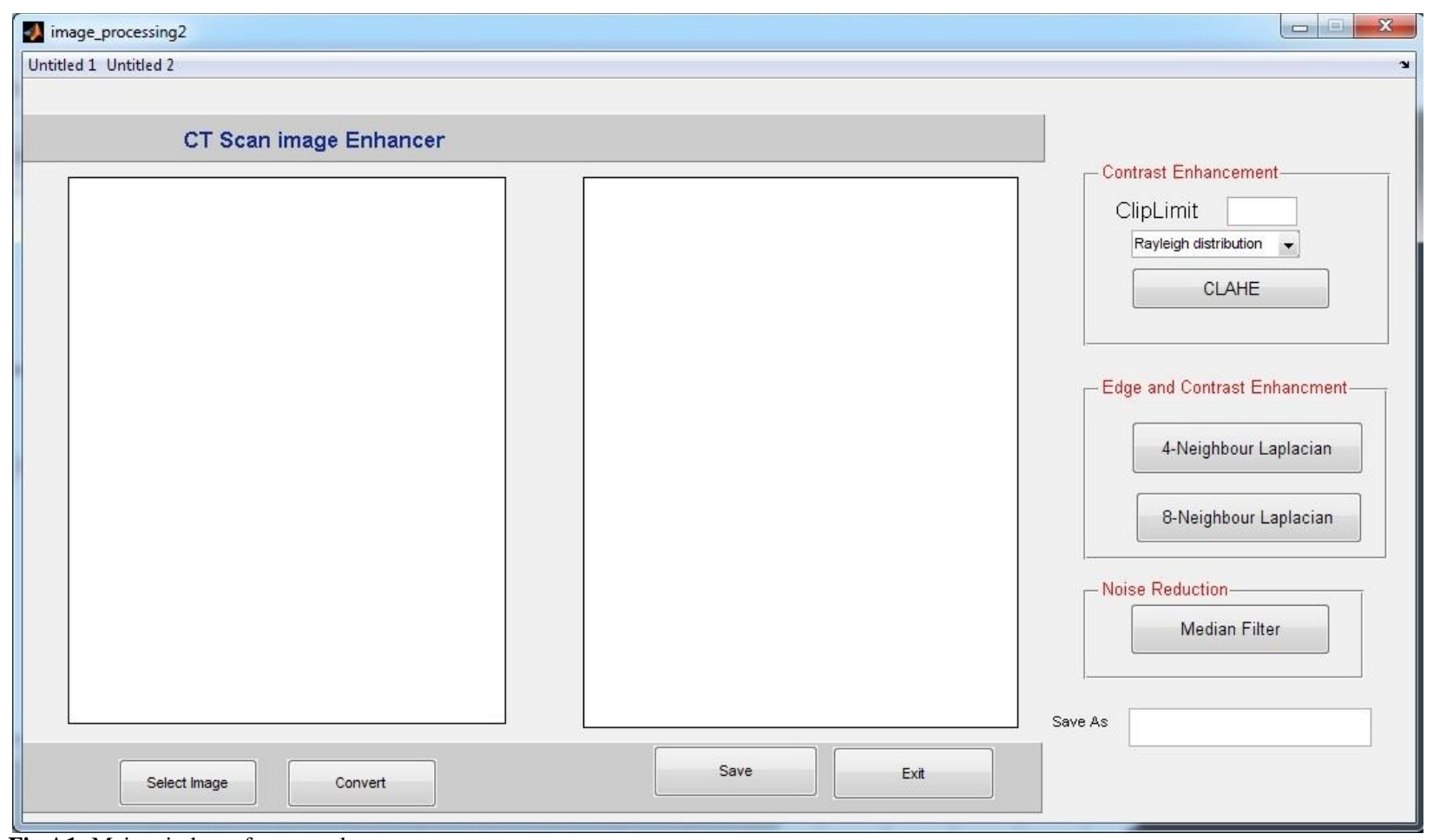

Fig A1: Main window of proposed system 